\title{
Network Utility Maximization under Maximum Delay Constraints and Throughput Requirements
}

\author{
Qingyu Liu, Haibo Zeng \\ Electrical and Computer Engineering \\ Virginia Tech
}

\author{
Minghua Chen \\ Information Engineering \\ The Chinese University of Hong Kong
}

\begin{abstract}
We consider the problem of maximizing aggregate user utilities over a multi-hop network, subject to link capacity constraints, maximum end-to-end delay constraints, and user throughput requirements. A user's utility is a concave function of the achieved throughput or the experienced maximum delay. The problem is important for supporting real-time multimedia traffic, and is uniquely challenging due to the need of simultaneously considering maximum delay constraints and throughput requirements. We first show that it is NP-complete either (i) to construct a feasible solution strictly meeting all constraints, or (ii) to obtain an optimal solution after we relax maximum delay constraints or throughput requirements up to constant ratios. We then develop a polynomial-time approximation algorithm named PASS. The design of PASS leverages a novel understanding between non-convex maximum-delay-aware problems and their convex average-delay-aware counterparts, which can be of independent interest and suggest a new avenue for solving maximum-delay-aware network optimization problems. Under realistic conditions, PASS achieves constant or problem-dependent approximation ratios, at the cost of violating maximum delay constraints or throughput requirements by up to constant or problemdependent ratios. PASS is practically useful since the conditions for PASS are satisfied in many popular application scenarios. We empirically evaluate PASS using extensive simulations of supporting video-conferencing traffic across Amazon EC2 datacenters. Compared to existing algorithms and a conceivable baseline, PASS obtains up to $100 \%$ improvement of utilities, by meeting the throughput requirements but relaxing the maximum delay constraints that are acceptable for practical video conferencing applications.
\end{abstract}

\section{CCS CONCEPTS}

- Mathematics of computing $\rightarrow$ Network flows; • Networks $\rightarrow$ Network resources allocation;

\section{KEYWORDS}

Network utility maximization, multiple-unicast network flow, delayaware network optimization

\section{ACM Reference Format:}

Qingyu Liu, Haibo Zeng and Minghua Chen. 2018. Network Utility Maximization under Maximum Delay Constraints and Throughput Requirements.

Permission to make digital or hard copies of all or part of this work for personal or classroom use is granted without fee provided that copies are not made or distributed for profit or commercial advantage and that copies bear this notice and the full citation on the first page. Copyrights for components of this work owned by others than ACM must be honored. Abstracting with credit is permitted. To copy otherwise, or republish, to post on servers or to redistribute to lists, requires prior specific permission and/or a fee. Request permissions from permissions@acm.org.

Submission to MobiHoc '19, fuly 2-5, 2019, Catania, Italy

(c) 2018 Association for Computing Machinery.

ACM ISBN x...\$15.00

https://doi.org/x
In Submission to MobiHoc '19, July 2-5, 2019, Catania, Italy. ACM, New York, NY, USA, 11 pages. https://doi.org/x

\section{INTRODUCTION}

We consider a multiple-unicast communication scenario where each unicast source streams a network flow to its destination over a multi-hop network, possibly using multiple paths. We study the problem of maximizing aggregate user utilities, subject to link capacity constraints, maximum delay constraints, and user throughput requirements. A user's utility is a concave function of the achieved throughput or the experienced maximum delay. The maximum delay denotes the maximum Source-to-Destination (S2D) delay, or equivalently the delay of the slowest S2D path that carries traffic.

Our study is motivated by the increasingly interests on supporting delay-critical traffic in various applications, e.g., video conferencing $[2,9,16]$. It is reported that 51 million users per month attend WebEx meetings, and 3 billion minutes of calls per day use Skype [15]. Low S2D delay is vital for such video conferencing applications. As recommended by the International Telecommunication Union (ITU) [12], a delay less than $150 \mathrm{~ms}$ can provide a transparent interactivity while delays above $400 \mathrm{~ms}$ are unacceptable for video conferencing. We remark that the maximum S2D delay, instead of the average one, is a critical concern for provisioning low delay services, since there may exist traffic which experiences an arbitrarily large S2D delay even for the solution that minimizes average S2D delay performance [15]. In sharp contrast, all the traffic can be streamed from its source to its destination timely following any solution that has an acceptable maximum S2D delay performance, because the maximum S2D delay is defined as an upper bound of S2D delays of all the traffic.

We consider a delay model where transmission over a link experiences a constant delay if the aggregate flow rate of the link is within a constant capacity, and unbounded delay otherwise. This model fits a number of practical applications, particularly the routing of delay-critical video conferencing traffic over interdatacenter networks. Specifically, according to recent reports from Microsoft [10] and Google [13], most real-world inter-datacenter networks are characterized by sharing link bandwidth for different applications, with over-provisioned link capacities. (i) Real-world inter-datacenter networks nowadays are utilized to simultaneously support traffic from various services, some of which have stringent delay requirements (e.g., video conferencing) while others are bandwidth-hungry and less sensitive to delay (e.g., data maintenance). Link capacity is often reserved separately for different types of services depending on their characteristics. (ii) Cloud providers typically over-provision inter-datacenter link capacity by $2-3$ times on a dedicated backbone to guarantee reliability, and the average link-capacity utilizations (the aggregate utilization of applications, not the bandwidth-utilization of individual applications) for busy 
Table 1: Compare our work with existing studies.

\begin{tabular}{|c|c|c|c|c|c|}
\hline & \multicolumn{2}{|c|}{ Maximization Objective } & \multicolumn{2}{|c|}{ Constraints } & Networking Setting \\
\hline & $\begin{array}{c}\text { Aggregate Throughput- } \\
\text { Based Utilities }\end{array}$ & $\begin{array}{c}\text { Aggregate Maximum- } \\
\text { Delay-Based Utilities }\end{array}$ & $\begin{array}{c}\text { Throughput } \\
\text { Requirements }\end{array}$ & $\begin{array}{c}\text { Maximum Delay } \\
\text { Constraints }\end{array}$ & Multiple-Unicast \\
\hline Many, e.g., $[14,17,19,21]$ & $\sqrt{ }$ & $x$ & $\checkmark$ & $x$ & $\checkmark$ \\
\hline$[3,4,15,18,26]$ & $x$ & $\boldsymbol{V}^{*}$ & $\checkmark$ & $x$ & $x$ \\
\hline$[1,25]$ & $\boldsymbol{V}^{* *}$ & $x$ & $x$ & $\sqrt{ }$ & $\sqrt{ }$ \\
\hline Out Work & $\checkmark$ & $\sqrt{ }$ & $\checkmark$ & $\checkmark$ & $\checkmark$ \\
\hline
\end{tabular}

**: The objective of $[1,25]$ is to maximize throughput, which is a special case of maximizing throughput-based utility functions.

links are $30-60 \%$ [16]. As such, for applications whose traffic volume is within the reserved capacity for their types of service, queuing delays are negligible and the constant propagation delays dominate end-to-end delays, as evaluated by [16] in a realistic network of Amazon EC2. Otherwise, if the traffic volume exceeds the reserved capacity, the applications will start to experience substantial queuing delays and thus substantial end-to-end delays. These observations justify our link capacity and delay model, especially for the critical problem of routing video-conferencing traffic over real-world inter-datacenter networks.

\subsection{Existing Studies}

We summarize existing studies in Tab. 1. In the literature, there exist many network utility maximization studies with throughput concerns, e.g., $[14,17,19,21]$, but less of them consider maximum delays. This is because the maximum delay of a single-unicast network flow is non-convex with the flow decision variables, and hence even a maximum-delay-aware problem in a simple networking scenario, e.g., the single-unicast maximum delay minimization problem, is NP-hard and thus challenging to solve [18].

Misra et al. [18] study the single-unicast maximum delay minimization problem subject to a throughput requirement, and design a Fully-Polynomial-Time Approximation Scheme (FPTAS). Zhang et al. [26] generalize the FPTAS of [18] and develop an FPTAS to minimize maximum delay subject to throughput, reliability, and differential delay constraints also in the single-unicast scenario. We observe that both FPTASes require to solve flow problems iteratively in time-expanded networks, by employing a binary-search based idea applicable only in the single-unicast setting. It is thus unclear how to extend their techniques to the general multiple-unicast scenario where the utility of an unicast (user) can be a concave function with the experienced maximum delay.

Cao et al. [1] develop an FPTAS that can maximize throughputs subject to maximum delay constraints in a multiple-unicast setting. This FPTAS is generalized by Yu et al. [25] to design FPTASes for other throughput maximization problems for practical IoT applications. Similar to FPTASes proposed by $[18,26]$, to satisfy maximum delay constraints while optimizing throughputs, FPTASes of [1, 25] require to solve flow problems iteratively in time-expanded networks, which is time-consuming. Moreover, the design of FPTASes in $[1,25]$ leverages the primal-dual algorithm, where their primal problems and associated dual problems need to be casted as linear programs. It is unclear how to extend their technique to the general scenario where the utility of an unicast can be a concave function with the achieved throughput.
We note that there exist other maximum-delay-aware studies in the literature. However, they only develop heuristic approaches instead of approximation algorithms. For example, Liu et al. [16] target the multicast maximum delay optimization problems. Their heuristic approach suffers from two limitations: (i) the running time could be high because the number of variables increases exponentially in the network size, and (ii) there is not yet theoretical performance guarantee of the achieved solution.

Instead of modeling link delay as a constant within a capacity as in $[1,16,18,25,26]$, there exist studies which model the link delay as a link-flow-dependent function. For example, Correa et al. $[3,4]$ minimize maximum delay with delay-function-dependent approximation ratios guaranteed. Liu et al. [15] minimize maximum delay with constant approximation ratios guaranteed. Our study models link delay as a constant within a capacity, which is the same as those in $[1,16,18,25,26]$, but different from the ones in $[3,4,15]$. We remark that maximum-delay-aware problems are fundamentally different with these different link delay models, since it is APX-hard to minimize the single-unicast maximum delay (hence no PTAS exists unless $\mathrm{P}=\mathrm{NP}$ ) with the flow-dependent delay model [4], but an FPTAS $^{1}$ exists to minimize the single-unicast maximum delay with the constant delay model [18].

Overall, with the constant delay model, existing maximum-delayaware studies focus on either the throughput-constrained maximum delay minimization problem or the maximum-delay-constrained throughput maximization problem, which are just special cases of our problem (Tab. 1). To design approximation algorithms, they rely on a technique of solving problems in expanded networks iteratively, leading to impractically high time complexities (e.g., at least $O\left(|E|^{3}|V|^{4} \mathcal{L}\right)$ to minimize single-unicast maximum delay where $|V|$ is number of nodes, $|E|$ is number of links, and $\mathcal{L}$ is input size of the given problem instance [18]). It is unclear how to generalize their techniques to our multiple-unicast utility maximization scenario, where the utility of an unicast is a concave function of the achieved throughput or the experienced maximum delay. In sharp contrast, we develop an approximation algorithm for our problem of maximizing utilities, by leveraging a novel understanding between non-convex maximum-delay-aware problems and their convex average-delay-aware counterparts. Specifically, we solve an average-delay-aware problem only once in the input network, and then deletes certain flow rate from individual unicast flows, resulting in a small time complexity (e.g., $O\left(|E|^{3} \mathcal{L}\right)$ to minimize single-unicast maximum delay in a dense network (Thm. 3.2).

\footnotetext{
${ }^{1}$ Unless $\mathrm{P}=\mathrm{NP}$, it holds that FPTAS $\subset$ PTAS in that the runtime of a PTAS is required to be polynomial in problem input but not $1 / \epsilon$, while the runtime of an FPTAS is polynomial in both the problem input and $1 / \epsilon$ [23].
} 
Network Utility Maximization under Maximum Delay Constraints and Throughput Requirements

\subsection{Our Contributions}

In this paper, we study a multiple-unicast flow problem of maximizing aggregate user utilities over a multi-hop network, subject to link capacity constraints, maximum delay constraints, and user throughput requirements. We make the following contributions.

$\triangleright$ We prove that it is NP-complete either (i) to construct a feasible solution meeting all constraints, or (ii) to obtain an optimal solution after we relax maximum delay constraints or throughput requirements up to constant ratios, due to the need of simultaneously considering maximum delay constraints and user throughput requirements.

$\triangleright$ We design an algorithm named PASS (Polynomial-time Algorithm Supporting utility-maximal flows Subject to throughput/delay constraints) for constructing approximate solutions to our problem in a polynomial time. Our design leverages a novel understanding between non-convex maximum-delay-aware problems and their convex average-delay-aware counterparts, which can be of independent interest and suggests a new avenue for solving maximumdelay-aware network optimization problems.

$\triangleright$ We characterize sufficient conditions for PASS to solve our problem in a polynomial time, providing (i) a constant approximation ratio after relaxing throughput requirements and maximum delay constraints by constant ratios, or (ii) a problem-dependent approximation ratio satisfying maximum delay constraints, after relaxing throughput requirements by a problem-dependent ratio, or (iii) a problem-dependent approximation ratio satisfying throughput requirements, after relaxing maximum delay constraints by a problem-dependent ratio. We note that one can use pre-scaled maximum delay constraints or throughput requirements as the input to PASS to generate feasible solutions as the output.

$\triangle$ We observe that our characterized conditions are satisfied in many popular application settings, where PASS can be applied with strong theoretical performance guarantee. Representative settings include minimizing throughput-constrained maximum delay and maximizing maximum-delay-constrained network utility. We evaluate the empirical performance of PASS in simulations of supporting video-conferencing traffic across Amazon EC2 datacenters. Compared to existing algorithms as well as a conceivable baseline, PASS can obtain up to $100 \%$ improvement of utilities, by meeting throughput requirements but relaxing maximum delay constraints that are acceptable for video conferencing applications.

\section{SYSTEM MODEL}

\subsection{Preliminary}

We consider a multi-hop network modeled as a directed graph $G \triangleq(V, E)$ with $|V|$ nodes and $|E|$ links. Each link $e \in E$ has a constant capacity $c_{e} \geq 0$ and a constant delay $d_{e} \geq 0$. For each link $e \in E$, data streamed to $e$ experiences a delay of $d_{e}$ to pass it, and the rate of streaming data to $e$ must be within the capacity $c_{e}$. We are given $K$ users, where for each user $i(i=1,2, \ldots, K)$, a source $s_{i} \in V$ needs to stream a single-unicast network flow to a destination $t_{i} \in V \backslash\left\{s_{i}\right\}$, possibly using multiple paths.

We denote $P_{i}$ as the set of all simple paths from $s_{i}$ to $t_{i}$, and $P \triangleq \cup_{i=1}^{K} P_{i}$. For any $p \in P$, its path delay $d^{p}$ is defined as

$$
d^{p} \triangleq \sum_{e \in E: e \in p} d_{e},
$$

\section{Submission to MobiHoc '19, July 2-5, 2019, Catania, Italy}

i.e., the summation of link delays along the path. We denote a multiple-unicast network flow solution as $f \triangleq\left\{f_{i}, i=1,2, \ldots, K\right\}$, where a single-unicast flow $f_{i}$ is defined as the assigned flow rate over $P_{i}$, i.e., $f_{i} \triangleq\left\{x^{p}: x^{p} \geq 0, p \in P_{i}\right\}$. For $f_{i}$, we define

$$
x_{i}^{e} \triangleq \sum_{p \in P_{i}: e \in p} x^{p}
$$

as the aggregated link rate of $e \in E$ of the unicast $i$ (or the user $i$ equivalently). Similarly, we denote $x_{e}$ as the total aggregated link rate of link $e \in E$, and

$$
x_{e} \triangleq \sum_{i=1}^{K} x_{i}^{e}=\sum_{p \in P: e \in p} x^{p} .
$$

We further denote the flow rate, or the throughput equivalently, achieved by a single-unicast flow $f_{i}$ by $\left|f_{i}\right|$,

$$
\left|f_{i}\right| \triangleq \sum_{p \in P_{i}} x^{p}=\sum_{e \in \operatorname{Out}\left(s_{i}\right)} x_{i}^{e}=\sum_{e \in \ln \left(t_{i}\right)} x_{i}^{e},
$$

where $\operatorname{Out}(v)($ resp. $\operatorname{In}(\mathrm{v}))$ is the set of outgoing (resp. incoming) links of $v$. The maximum delay experienced by $f_{i}$ is defined as

$$
\mathcal{M}\left(f_{i}\right) \triangleq \max _{p \in P_{i}: x^{p}>0} d^{p},
$$

i.e., the delay of the longest (slowest) path with positive rates from $s_{i}$ to $t_{i}{ }^{2}$. The total delay of $f_{i}$ is defined as

$$
\mathcal{T}\left(f_{i}\right) \triangleq \sum_{p \in P_{i}}\left(x^{p} \cdot d^{p}\right)=\sum_{e \in E}\left(x_{i}^{e} \cdot d_{e}\right) .
$$

With $\mathcal{T}\left(f_{i}\right)$, we can easily define the average delay experienced by $f_{i}$ as $\mathcal{A}\left(f_{i}\right) \triangleq \mathcal{T}\left(f_{i}\right) /\left|f_{i}\right|$, and we let $\mathcal{A}\left(f_{i}\right)=0$ if $\left|f_{i}\right|=0$.

For each $f_{i}, i=1,2, \ldots, K$, we denote its throughput-based utility as $\mathcal{U}_{i}^{t}\left(\left|f_{i}\right|\right)$, which is a function that rewards $f_{i}$ based on the achieved throughput. Similarly, we denote its maximum-delaybased utility as $-\mathcal{U}_{i}^{d}\left(\mathcal{M}\left(f_{i}\right)\right)$, where $\mathcal{U}_{i}^{d}\left(\mathcal{M}\left(f_{i}\right)\right)$ is a function that penalizes $f_{i}$ based on the experienced maximum delay.

\subsection{Problem Definition}

In this paper, we study the following problem of Maximizing aggregate user Utilities subject to link capacity constraints, maximum Delay constraints, and Throughput requirements (MUDT),

$$
\begin{array}{cl}
\text { (MUDT) : obj: } \quad \text { either } \max \sum_{i=1}^{K} \mathcal{U}_{i}^{t}\left(\left|f_{i}\right|\right), \\
& \text { or } \max -\sum_{i=1}^{K} \mathcal{U}_{i}^{d}\left(\mathcal{M}\left(f_{i}\right)\right), \\
\text { s.t. } \quad & \left|f_{i}\right| \geq R_{i}, \forall i=1,2, \ldots, K, \\
& \mathcal{M}\left(f_{i}\right) \leq D_{i}, \forall i=1,2, \ldots, K, \\
& f=\left\{f_{1}, f_{2}, \ldots, f_{K}\right\} \in \mathcal{X},
\end{array}
$$

where $\mathcal{X}$ defines a feasible multiple-unicast flow $f$ meeting flow conservation constraints and link capacity constraints, i.e.,

$$
\begin{aligned}
& \mathcal{X} \triangleq\left\{\sum_{e \in \operatorname{Out}\left(s_{i}\right)} x_{i}^{e}=\sum_{e \in \ln \left(t_{i}\right)} x_{i}^{e}=\left|f_{i}\right|, \forall 1 \leq i \leq K,\right. \\
& \quad \sum_{e \in \operatorname{Out}(v)} x_{i}^{e}=\sum_{e \in \ln (v)} x_{i}^{e}, \forall v \in V \backslash\left\{s_{i}, t_{i}\right\}, \forall 1 \leq i \leq K, \\
& \overline{{ }^{2} \text { We call a path } p} \in P_{i} \text { with } x^{p}>0 \text { as a flow-carrying path of } f_{i} .
\end{aligned}
$$




$$
\left.\sum_{i=1}^{K} x_{i}^{e} \leq c_{e}, \forall e \in E, \text { vars: } x_{i}^{e} \geq 0, \forall e \in E, \forall 1 \leq i \leq K\right\} .
$$

In formula (1), the objective (1a) (resp. (1b)) maximizes the aggregate throughput-based utilities (resp. maximum-delay-based utilities) of all the users, the throughput requirements (1c) require the throughput achieved by each user $i$ to be no smaller than $R_{i}$, the maximum delay constraints $(1 \mathrm{~d})$ restrict the maximum delay experienced by each user $i$ to be no greater than $D_{i}$, and the feasibility constraint (1e) defines a feasible multiple-unicast network flow solution, meeting link capacity constraints.

In the end of this section, we give an important theorem of MUDT, which argues that it is impossible even to (i) construct a feasible solution meeting all constraints, or (ii) obtain an optimal solution meeting relaxed constraints, in a polynomial time, unless $\mathrm{P}=\mathrm{NP}$. Thus it is non-trivial to develop polynomial-time approximation algorithms for MUDT subject to relaxed constraints.

Theorem 2.1. For MUDT, it is NP-complete (i) to construct a feasible solution that meets all constraints, or (ii) to obtain an optimal solution that meets throughput requirements but relaxes maximum delay constraints, or (iii) to obtain an optimal solution that meets maximum delay constraints but relaxes throughput requirements.

Proof. Refer to our Appendix 7.3.

\section{PROPOSED ALGORITHM PASS}

In this section we design an algorithm PASS for MUDT of maximizing aggregate user utilities. We characterize conditions of the input utility functions such that PASS theoretically gives approximate solutions in a polynomial time, meeting relaxed constraints.

\subsection{Algorithmic Structure of PASS}

We note that the non-convex maximum delays bring difficulties for solving MUDT. The key idea of our proposed PASS is to replace the non-convex maximum delays in MUDT by the convex average delays, and solve the average-delay-aware counterpart to obtain an approximate solution to MUDT in a polynomial time. (i) We denote the average-delay-aware counterpart of the MUDT that maximizes throughput-based utilities, i.e., problem (1) with an objective of (1a), as MUAT-T, with the following formulation

$$
\begin{aligned}
\text { (MUAT-T) : obj: } \quad & \max \sum_{i=1}^{K} \mathcal{U}_{i}^{t}\left(\left|f_{i}\right|\right), \\
\text { s.t. } \quad & \left|f_{i}\right| \geq R_{i}, \forall i=1,2, \ldots, K, \\
& \mathcal{T}\left(f_{i}\right) \leq D_{i} \cdot\left|f_{i}\right|, \forall i=1,2, \ldots, K, \\
& f=\left\{f_{1}, f_{2}, \ldots, f_{K}\right\} \in \mathcal{X} .
\end{aligned}
$$

(ii) Similarly, we denote the average-delay-aware counterpart of the MUDT that maximizes maximum-delay-based utilities, i.e., problem (1) with an objective of (1b), as MUAT-M. MUAT-M has the following formulation

$$
\begin{aligned}
\text { (MUAT-M ) : obj: } \quad & \max -\sum_{i=1}^{K} \mathcal{U}_{i}^{d}\left(\frac{\mathcal{T}\left(f_{i}\right)}{R_{i}}\right), \\
\text { s.t. } & \left|f_{i}\right|=R_{i}, \forall i=1,2, \ldots, K, \\
& \mathcal{T}\left(f_{i}\right) \leq D_{i} \cdot R_{i}, \forall i=1,2, \ldots, K, \\
& f=\left\{f_{1}, f_{2}, \ldots, f_{K}\right\} \in \mathcal{X} .
\end{aligned}
$$

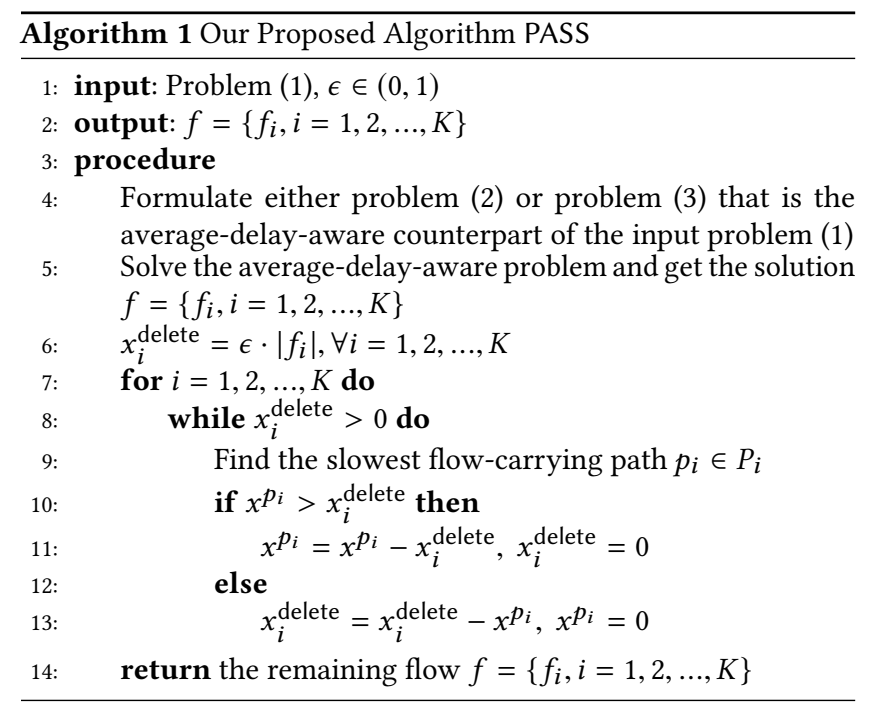

Algorithm 1 describes the details of PASS. It first solves the average-delay-aware counterpart of the MUDT and obtain the corresponding multiple-unicast flow solution $f=\left\{f_{i}, i=1,2, \ldots, K\right\}$ (line 5). Next for each $i=1,2, \ldots, K$, we delete a rate of $\epsilon \cdot\left|f_{i}\right|$ iteratively from the slowest flow-carrying paths of $f_{i}$ (line 8 ). In the end, the remaining flow is the solution returned by PASS.

\subsection{PASS can Solve MUDT Approximately, Meeting Relaxed Constraints}

Now we give an important lemma which will be used later to prove the approximation ratio of our PASS.

Lemma 3.1. In Algorithm 1 with an arbitrary $\epsilon \in(0,1)$, suppose $\hat{f}=\left\{\hat{f}_{i}, i=1,2, \ldots, K\right\}$ is the solution to the average-delay-aware counterpart of MUDT (solution achieved in line 5), and suppose $\bar{f}=$ $\left\{\bar{f}_{i}, i=1,2, \ldots, K\right\}$ is the solution returned in the end (the remaining solution achieved in line 14$)$. For any $i=1,2, \ldots, K$, we have

$$
\mathcal{T}\left(\bar{f}_{i}\right)+\epsilon \cdot\left|\hat{f}_{i}\right| \cdot \mathcal{M}\left(\bar{f}_{i}\right) \leq \mathcal{T}\left(\hat{f}_{i}\right) .
$$

Proof. Refer to our Appendix 7.1.

Lem. 3.1 implies that $\epsilon \cdot \mathcal{M}\left(\bar{f}_{i}\right) \leq \mathcal{A}\left(\hat{f}_{i}\right), \forall i=1,2, \ldots, K$, i.e., the maximum delay of each single-unicast flow after deleting rate is bounded by a constant ratio as compared to the average delay of the corresponding single-unicast flow before deleting rate. With this critical observation that relates the non-convex maximum delays with the convex average delays, we can characterize conditions for PASS to solve MUDT approximately in a polynomial time.

THeOREm 3.2. Given a feasible problem (1), suppose we use PASS (Algorithm 1) with an arbitrary $\epsilon \in(0,1)$ to solve it. If the problem is feasible, meeting all conditions below

(1) for each $i=1,2, \ldots, K$, for an arbitrary $a \geq 0, \mathcal{U}_{i}^{t}(a)$ is concave, non-decreasing, and non-negative with $a, \mathcal{U}_{i}^{d}(a)$ is convex, non-decreasing, and non-negative with $a$,

(2) for an arbitrary $a \geq 0$, the following holds given any $\sigma \geq 1$

$$
\mathcal{U}_{i}^{d}(\sigma \cdot a) \leq \sigma \cdot \mathcal{U}_{i}^{d}(a), \forall i=1,2, \ldots, K,
$$


Network Utility Maximization under Maximum Delay Constraints and Throughput Requirements

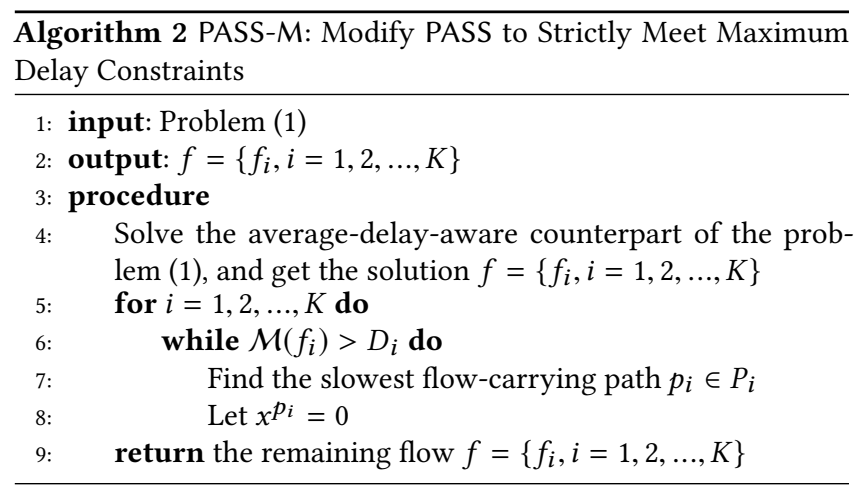

then PASS must return a solution $\bar{f}=\left\{\bar{f}_{i}, i=1, \ldots, K\right\}$ in a polynomial time, meeting the following relaxed constraints

$$
\begin{aligned}
& \left|\bar{f}_{i}\right| \geq(1-\epsilon) \cdot R_{i}, \forall i=1,2, \ldots, K, \\
& \mathcal{M}\left(\bar{f}_{i}\right) \leq D_{i} / \epsilon, \forall i=1,2, \ldots, K, \\
& \bar{f}=\left\{\bar{f}_{1}, \bar{f}_{2}, \ldots, \bar{f}_{K}\right\} \in \mathcal{X} .
\end{aligned}
$$

Suppose $f^{*}=\left\{f_{i}^{*}, i=1,2, \ldots, K\right\}$ is the optimal solution to the problem (1). If the throughput-based utility maximization (1a) is the objective, $\bar{f}$ provides the following approximation ratio

$$
\sum_{i=1}^{K} \mathcal{U}_{i}^{t}\left(\left|\bar{f}_{i}\right|\right) \geq(1-\epsilon) \cdot \sum_{i=1}^{K} \mathcal{U}_{i}^{t}\left(\left|f_{i}^{*}\right|\right)
$$

If the maximum-delay-based utility maximization (1b) is the objective, $\bar{f}$ provides the following approximation ratio

$$
\sum_{i=1}^{K} \mathcal{U}_{i}^{d}\left(\mathcal{M}\left(\bar{f}_{i}\right)\right) \leq \frac{1}{\epsilon} \cdot \sum_{i=1}^{K} \mathcal{U}_{i}^{d}\left(\mathcal{M}\left(f_{i}^{*}\right)\right) .
$$

Proof. Refer to our Appendix 7.2.

It is clear that PASS provides a constant approximation ratio, at the cost of violating throughput requirements (1c) by a constant ratio of $(1-\epsilon)$, and violating maximum delay constraints (1d) by a constant ratio of $1 / \epsilon$. For certain applications, the throughput requirements or the maximum delay constraints are hard constraints that cannot be violated. We note that one can use pre-scaled maximum delay constraints and throughput requirements as the input to PASS to generate feasible solutions as the output. Moreover, in the following, by slightly modifying PASS, we respectively develop (i) an algorithm PASS-M to achieve approximate solutions that can strictly meet maximum delay constraints, and (ii) an algorithm PASS-T to achieve approximate solutions that can strictly meet throughput requirements.

\subsection{Modify PASS to Strictly Meet Maximum Delay Constraints}

We introduce PASS-M in Algorithm 2. Similar to PASS, PASS-M first solves the average-delay-aware counterpart of MUDT. But different from PASS that deletes $\epsilon \cdot\left|f_{i}\right|$ rate from slowest flow-carrying paths of each $f_{i}$, PASS-M deletes rate from slowest flow-carrying paths of $f_{i}$ till the maximum delay of $f_{i}$ strictly meets the constraint $D_{i}$. In the following theorem, we prove that PASS-M can obtain a solution with a problem-dependent approximation ratio.
Submission to MobiHoc '19, July 2-5, 2019, Catania, Italy

THeOREM 3.3. Given a feasible problem (1), suppose it meets all conditions in Thm. 3.2. Suppose we use PASS-M (Algorithm 2) to solve it. Then PASS-M must return a solution $\bar{f}=\left\{\bar{f}_{i}, i=1,2, \ldots, K\right\}$ in a polynomial time, meeting the following relaxed constraints

$$
\begin{aligned}
& \left|\bar{f}_{i}\right| \geq\left(1-\epsilon_{\max }\right) \cdot R_{i}, \forall i=1,2, \ldots, K, \\
& \mathcal{M}\left(\bar{f}_{i}\right) \leq D_{i}, \forall i=1,2, \ldots, K, \\
& \bar{f}=\left\{\bar{f}_{1}, \bar{f}_{2}, \ldots, \bar{f}_{K}\right\} \in X,
\end{aligned}
$$

where $\epsilon_{\max }$ is defined as follows

$$
\epsilon_{\max }=\max _{1 \leq i \leq K}\left\{\left(\left|\hat{f}_{i}\right|-\left|\bar{f}_{i}\right|\right) /\left|\hat{f}_{i}\right|\right\},
$$

where $\hat{f}=\left\{\hat{f}_{i}, i=1,2, \ldots, K\right\}$ is the optimal solution to the averagedelay-aware problem in line 4 of Algorithm 2. Suppose $f^{*}=\left\{f_{i}^{*}, i=\right.$ $1,2, \ldots, K\}$ is the optimal solution to problem (1). If the throughputbased utility maximization (1a) is the objective, $\bar{f}$ provides the following approximation ratio

$$
\sum_{i=1}^{K} \mathcal{U}_{i}^{t}\left(\left|\bar{f}_{i}\right|\right) \geq\left(1-\epsilon_{\max }\right) \cdot \sum_{i=1}^{K} \mathcal{U}_{i}^{t}\left(\left|f_{i}^{*}\right|\right) .
$$

If the maximum-delay-based utility maximization (1b) is the objective, $\bar{f}$ provides the following approximation ratio

$$
\sum_{i=1}^{K} \mathcal{U}_{i}^{d}\left(\mathcal{M}\left(\bar{f}_{i}\right)\right) \leq \frac{1}{\epsilon_{\min }} \cdot \sum_{i=1}^{K} \mathcal{U}_{i}^{d}\left(\mathcal{M}\left(f_{i}^{*}\right)\right),
$$

where $\epsilon_{\min }$ is defined as follows

$$
\epsilon_{\min }=\min _{1 \leq i \leq K}\left\{\left(\left|\hat{f}_{i}\right|-\left|\bar{f}_{i}\right|\right) /\left|\hat{f}_{i}\right|\right\} .
$$

Proof. Refer to our Appendix 7.4.

Comparing Thm. 3.2 of PASS with Thm. 3.3 of PASS-M, to solve MUDT, (i) PASS achieves a solution with a constant approximation ratio, at the cost of violating both throughput requirements and maximum delay constraints by constant ratios, while (ii) PASS-M obtains a solution with a problem-dependent approximation ratio, strictly meeting maximum delay constraints, but at the cost of violating throughput requirements by a problem-dependent ratio.

\subsection{Modify PASS to Strictly Meet Throughput Requirements}

In order to strictly meet throughput requirements, our PASS-T suggest to use the optimal solution to the average-delay-aware counterpart of MUDT directly as a solution to the maximum-delayaware problem MUDT, i.e.,

$\triangleright$ PASS-T: directly solve the average-delay-aware counterpart of the problem (1).

Theorem 3.4. Given a feasible problem (1), suppose it meets all conditions in Thm. 3.2. We denote $\bar{g}=\left\{\bar{g}_{1}, \bar{g}_{2}, \ldots, \bar{g}_{K}\right\}$ as the solution returned if we use PASS (Algorithm 1) to solve it with an $\epsilon \in(0,1)$. Now suppose we use PASS-T to solve the problem (1). Then PASS-T must return a solution $\bar{f}=\left\{\bar{f}_{i}, i=1,2, \ldots, K\right\}$ in a polynomial time, meeting the following relaxed constraints

$$
\begin{aligned}
& \left|\bar{f}_{i}\right| \geq R_{i}, \forall i=1,2, \ldots, K, \\
& \mathcal{M}\left(\bar{f}_{i}\right) \leq \frac{\lambda}{\epsilon} \cdot D_{i}, \forall i=1,2, \ldots, K, \\
& \bar{f}=\left\{\bar{f}_{1}, \bar{f}_{2}, \ldots, \bar{f}_{K}\right\} \in X,
\end{aligned}
$$


where $\lambda$ is defined as follows

$$
\lambda=\max \left\{1, \max _{1 \leq i \leq K}\left\{\mathcal{M}\left(\bar{f}_{i}\right) / \mathcal{M}\left(\bar{g}_{i}\right)\right\}\right\} .
$$

Suppose $f^{*}=\left\{f_{i}^{*}, i=1,2, \ldots, K\right\}$ is the optimal solution to problem (1). If the throughput-based utility maximization (1a) is the objective, $\bar{f}$ provides the following approximation ratio

$$
\sum_{i=1}^{K} \mathcal{U}_{i}^{t}\left(\left|\bar{f}_{i}\right|\right) \geq \sum_{i=1}^{K} \mathcal{U}_{i}^{t}\left(\left|f_{i}^{*}\right|\right)
$$

If the maximum-delay-based utility maximization (1b) is the objective, $\bar{f}$ provides the following approximation ratio

$$
\sum_{i=1}^{K} \mathcal{U}_{i}^{d}\left(\mathcal{M}\left(\bar{f}_{i}\right)\right) \leq \frac{\lambda}{\epsilon} \cdot \sum_{i=1}^{K} \mathcal{U}_{i}^{d}\left(\mathcal{M}\left(f_{i}^{*}\right)\right)
$$

Proof. Refer to our Appendix 7.5.

Thm. 3.4 suggests that we can figure out an approximation ratio of PASS-T with the knowledge of an arbitrary solution of PASS Comparing Thm. 3.2 of PASS with Thm. 3.4 of PASS-T, in order to solve MUDT, (i) PASS achieves a solution with a constant approximation ratio, at the cost of violating both throughput requirements and maximum delay constraints by constant ratios, while (ii) PASST obtains a solution with a problem-dependent approximation ratio, strictly meeting throughput requirements, but at the cost of violating maximum delay constraints by a problem-dependent ratio.

\subsection{Our Proposed Algorithms Can Solve Other Maximum-Delay-Aware Problems}

As shown in problem (1), MUDT has an objective of either (1a) or (1b), both of which maximize aggregate user utilities. Differently, another two representative user-utility-sensitive objectives are

$$
\begin{aligned}
& \max \min _{1 \leq i \leq K}\left\{\mathcal{U}_{i}^{t}\left(\left|f_{i}\right|\right)\right\}, \\
& \max \min _{1 \leq i \leq K}\left\{-\mathcal{U}_{i}^{d}\left(\mathcal{M}\left(f_{i}\right)\right)\right\},
\end{aligned}
$$

both of which maximize worst user utilities. Following same proof to Thm. 3.2, Thm. 3.3, and Thm. 3.4, it is easy to verify that as long as the conditions in Thm. 3.2 are satisfied, we can use PASS, PASS-M, and PASS-T to solve the problem with an objective of either (14a) or (14b), subject to throughput requirements (1c), maximum delay constraints (1d), and feasibility constraints (1e), approximately in a polynomial time. Our design of PASS suggests a new avenue for solving maximum-delay-aware network optimization problems.

Overall in this section, we design PASS to solve the maximumdelay-aware problem MUDT approximately in a polynomial time under practical conditions. PASS solves the average-delay-aware counterpart of MUDT only once in the input network, and then deletes certain flow rate from slowest flow-carrying paths to obtain solutions with theoretical performance guarantee. Note again that in sharp contrast, existing maximum-delay-aware problems either minimize throughput-constrained maximum delay or maximize maximum-delay-constrained throughput, which are special cases of our problem MUDT. They rely on a time-consuming technique of solving problems iteratively in the time-expanded network to provide approximate solutions. Our PASS leverages a novel understanding between non-convex maximum-delay-aware problems and their convex average-delay-aware counterparts, which can be of independent interest and suggest a new avenue for solving maximum-delay-aware network optimization problems.

\section{POPULAR DELAY-/THROUGHPUT- AWARE NETWORK COMMUNICATION SCENARIOS}

In this section we introduce several popular network communication settings that are sensitive both to the throughputs and to the maximum delays. Although associated problems are all NP-hard, we observe that they are all special cases of MUDT, and all satisfy conditions introduced in Thm. 3.2, and hence can be solved by PASS, PASS-M, and PASS-T approximately with strong theoretical performance guarantee in a polynomial time.

\subsection{Throughput-Constrained Maximum Delay Minimization}

The Throughput-Constrained maximum Delay Minimization problem (TCDM) aims to find a network flow to minimize the weighted summation of maximum delays of all users, subject to link capacity constraints and throughput requirements.

$$
\begin{aligned}
(\mathrm{TCD} M): \min & \sum_{i=1}^{K}\left(w_{i} \cdot \mathcal{M}\left(f_{i}\right)\right) \\
\text { s.t. } & \left|f_{i}\right| \geq R_{i}, \forall i=1,2, \ldots, K, \\
& f=\left\{f_{1}, f_{2}, \ldots, f_{K}\right\} \in \mathcal{X},
\end{aligned}
$$

where in the objective (15a) a non-negative weight $w_{i} \geq 0$ is associated with the maximum delay of $f_{i}$ for each $i=1,2, \ldots, K$.

TCDM is NP-hard, since as its special case when $K=1$, the singleunicast maximum delay minimization problem is known to be NPhard [18]. Maximum delay minimization problems similar to TCDM have been studied in $[3,4,15,18,26]$. It is clear that TCDM satisfies our conditions introduced in Thm. 3.2. Therefore, by replacing the non-convex maximum delays with the convex average delays, we can get the average-delay-aware counterpart formulated in the way of problem (3), and thus can either (i) use PASS to solve TCDM with a constant approximation ratio while violating throughput requirements also by a constant ratio (see Thm. 3.2), or (ii) use PASS-T to solve TCDM with a problem-dependent approximation ratio, strictly meeting throughput requirements (see Thm. 3.4).

\subsection{Maximum-Delay-Constrained Throughput-Based Utility Maximization}

The maximum-Delay-Constrained throughput-based Utility Maximization (DCUM) problem aims to find a network flow to maximize aggregate user utilities, subject to link capacity constraints and maximum delay constraints. It has the following formulation.

$$
\begin{aligned}
(\mathrm{DCUM}): \max & \sum_{i=1}^{K} \mathcal{U}_{i}^{t}\left(\left|f_{i}\right|\right) \\
\text { s.t. } & \mathcal{M}\left(f_{i}\right) \leq D_{i}, \forall i=1,2, \ldots, K, \\
& f=\left\{f_{1}, f_{2}, \ldots, f_{K}\right\} \in \mathcal{X} .
\end{aligned}
$$

DCUM is NP-hard, because as its special case when $K=1$ and $\mathcal{U}_{1}^{t}\left|f_{1}\right|=\left|f_{1}\right|$, the problem can be proved to be NP-hard following a similar proof as introduced in the Appendix of [18]. Throughputbased utility maximization problems similar to DCUM have been 
Network Utility Maximization under Maximum Delay Constraints and Throughput Requirements

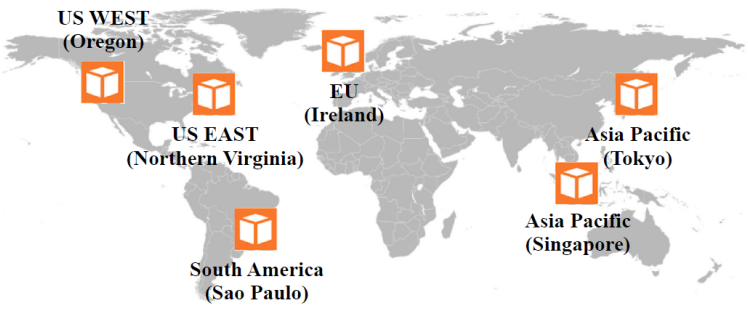

Figure 1: Topology of the 6 Amazon EC2 datacenters [16].

Table 2: Information of $\left(d_{e}, c_{e}\right)$ for each link $e \in E$ in the Amazon EC2 network $[9,16]$, where $d_{e}$ is link delay (in ms) and $c_{e}$ is link capacity (in Mbps), (OR: Oregon, VA: Virginia, IR: Ireland, TO: Tokyo, SI: Singapore, SP: Sao Paulo).

\begin{tabular}{|c|c|c|c|c|c|c|}
\hline & OR & VA & IR & TO & SI & SP \\
\hline OR & N/A & $(41,82)$ & $(86,86)$ & $(68,138)$ & $(117,74)$ & $(104,67)$ \\
\hline VA & - & N/A & $(54,72)$ & $(101,41)$ & $(127,52)$ & $(82,70)$ \\
\hline IR & - & - & N/A & $(138,56)$ & $(117,44)$ & $(120,61)$ \\
\hline TO & - & - & - & N/A & $(45,166)$ & $(151,41)$ \\
\hline SI & - & - & - & - & N/A & $(182,33)$ \\
\hline SP & - & - & - & - & - & N/A \\
\hline
\end{tabular}

studied in $[1,25]$. Due to practical concerns, it is fair to assume that the throughput-based utility function of each user is concave, non-decreasing, and non-negative with the achieved throughput, thus meeting conditions introduced in our Thm. 3.2. After replacing the non-convex maximum delays with the convex average delays, we can get the average-delay-aware counterpart formulated in the way of problem (2), and thus can either (i) use PASS to solve DCUM with a constant approximation ratio while violating maximum delay constraints also by a constant ratio (see Thm. 3.2), or (ii) use PASS$\mathrm{M}$ to solve DCUM with a problem-dependent approximation ratio, strictly meeting maximum delay constraints (see Thm. 3.3).

\section{PERFORMANCE EVALUATION}

We evaluate the empirical performance of our proposed algorithms, by simulating the delay-critical video conferencing traffic over a real-world continent-scale inter-datacenter network topology of 6 globally distributed Amazon EC2 datacenters (see Fig. 1). The network is modeled as a complete undirected graph. Each undirected link is treated as two directed links that operate independently and have identical delays and capacities, a common way to model an undirected graph by a directed one, e.g. in [7]. We set link delays and capacities according to practical evaluations on Amazon EC2 from $[9,16]$ (see Tab. 2). We assume two unicasts, namely $K=2$, with $s_{1}$ to be Virginia, $t_{1}$ to be Singapore, $s_{2}$ to be Oregon, and $t_{2}$ to be Tokyo. Our test environment is an Intel Core i5 $(2.40 \mathrm{GHz})$ processor with $8 \mathrm{~GB}$ memory running Windows 64-bit operating system. All the experiments are implemented in $\mathrm{C}++$ and linear programs are solved using CPLEX [11].

\subsection{Use PASS to Minimize Maximum Delay}

We now use PASS to minimize the maximum delay, subject to link capacity constraints and throughput requirements (i.e., to solve
Submission to MobiHoc '19, July 2-5, 2019, Catania, Italy
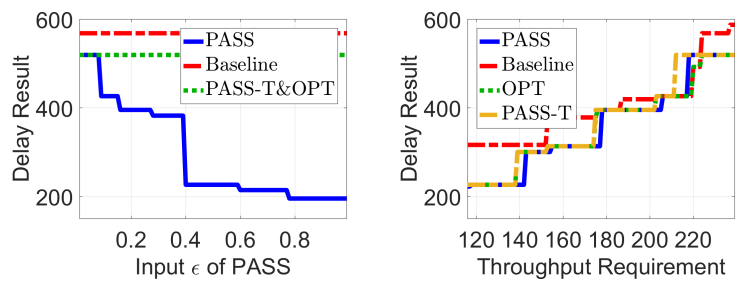

(a) Delay results with $\epsilon$ of PASS, with (b) Delay results with throughput re$R_{1}=R_{2}=230$. quirements, with $\epsilon=3 \%$ in PASS.

Figure 2: Simulation results of using PASS to minimize the summation of maximum delays.

TCDM with formula (15)). We assume $w_{1}=w_{2}$ and $R_{1}=R_{2}=R$ in the formula (15).

We compare PASS with the optimal solution, a conceivable greedy baseline, and PASS-T respectively. (i) Because link delays are all integers (see Tab. 2), the delay of any path must be an integer. Therefore, we can obtain the optimal solution minimizing the summation of maximum delays, by enumerating all possible maximum delays of individual unicasts to figure out the minimal performance such that a feasible flow exists in the time-expanded network. Note that this approach theoretically has an exponential time complexity, and is the foundation of the FPTAS [18] designed for the single-unicast maximum delay minimization problem. (ii) In order to minimize delay while satisfying throughput requirements, the baseline greedily obtains the routing solution from the unicast 1 to the unicast $K$ one by one. In the iteration of the unicast $i$, it assigns as much rate as possible to the shortest paths from $s_{i}$ to $t_{i}$ iteratively respecting the link capacity constraints, till the throughput requirement $R_{i}$ is satisfied. Similar heuristic approaches have been used in other delay-aware network flow studies, e.g., in [5].

First, we evaluate the summation of maximum delays of PASS with $\epsilon$ (see Fig. 2(a)). We set $R=230$ and vary $\epsilon$ from $1 \%$ to $99 \%$ by a step of $1 \%$. According to the figure, (i) PASS-T obtains the optimal solution to our problem, (ii) the delay of the baseline is strictly larger than optimal, and (iii) the delay of PASS is a staircase function with $\epsilon$. We remark that the delay of PASS can be smaller than optimal in many instances because PASS can only support $(1-\epsilon)$-fraction of the throughput requirement, while the optimal solution achieves the minimal summation of maximum delays among network flows supporting the full throughput requirement.

Second, we evaluate the summation of maximum delays of PASS with the throughput requirement $R$ (see Fig. 2(b)). We set $\epsilon=3 \%$ since a $3 \%$ throughput loss is very acceptable for video conferencing with protection/recovery capabilities [22]. We vary $R$ from 116 to 239 with a unit step. We remark that $116 \mathrm{Mbps}$ is the smallest throughput when the baseline needs multiple paths to forward it for each of the two unicasts, and 239Mbps is the largest throughput that can be routed. From Fig. 2(b), it is clear that PASS outputs a smaller maximum delay compared with the baseline in most instances. In average, the maximum delay of the baseline (402) is over $11 \%$ more than that of the optimal (362) and of the PASS (359). In the worst case $(R \in[116,138])$, the maximum delay of the baseline is over $40 \%$ 

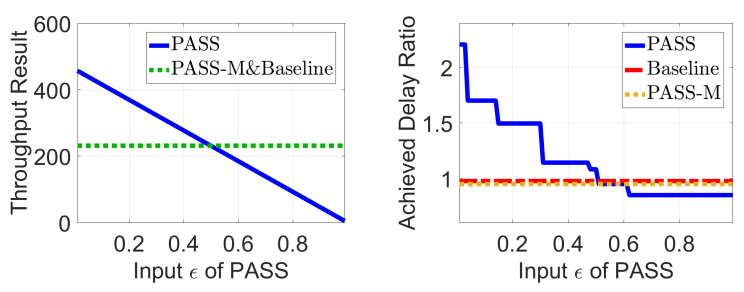

(a) Throughput results (both baseline (b) Delay ratio comparing the achieved and PASS- $M$ obtain the optimal). result to the constraint.

Figure 3: Simulation results of using PASS to maximize total throughput with various $\epsilon$, where $D_{1}=D_{2}=150$.

more than that of the optimal and of the PASS. In addition, PASS$T$ obtains the optimal solution to our problem in most instances, except for instances where $R \in[212,223]$.

\subsection{Use PASS to Maximize Throughput}

We then use PASS to maximize the throughput, subject to link capacity constraints and maximum delay constraints (i.e., to solve DCUM with formula (16)). We assume $\mathcal{U}_{1}^{t}\left(\left|f_{1}\right|\right)=\left|f_{1}\right|, \mathcal{U}_{2}^{t}\left(\left|f_{2}\right|\right)=\left|f_{2}\right|$, and $D_{1}=D_{2}=D$ in the formula (16). We compare PASS with the optimal solution, a conceivable baseline, and PASS-M, respectively. Similar to the greedy approach introduced in Sec. 5.1, the baseline assigns as much rate as possible to the shortest paths respecting both link capacity constraints and maximum delay constraints iteratively from the unicast 1 to the unicast $K$ one by one. Besides, similar to Sec. 5.1, we can obtain the optimal solution maximizing throughput by solving multiple-unicast flow problems in the time-expanded network.

We set $D=150$ due to the following two concerns. (i) An end-toend delay less than $150 \mathrm{~ms}$ can provide a transparent interactivity for video conferencing [12]. (ii) A delay larger than $150 \mathrm{~ms}$ (as long as it is less than 400ms) is still acceptable for video conferencing [12], and hence a solution that violates the maximum delay constraint (e.g., the solution of PASS) may still be useful if it can achieve a huge amount of throughput increment.

We vary $\epsilon$ from $1 \%$ to $99 \%$ with a step of $1 \%$. We give the throughput results in Fig. 3(a), and the achieved maximum delay ratio results, i.e., $\max \left\{\mathcal{M}\left(f_{1}\right), \mathcal{M}\left(f_{2}\right)\right\} / D$ where $f$ is the solution, in Fig. 3(b). In our simulations, both the baseline and PASS-M obtain the optimal throughput strictly meeting maximum delay constraints. For $\epsilon \leq 49 \%$, the throughput of PASS is strictly larger than the optimal, while violating maximum delay constraints (e.g., $8 \%$ more than $D$ when $\epsilon=49 \%$ ). For $\epsilon \geq 51 \%$, the solution of PASS meets maximum delay constraints, but the achieved throughput is strictly smaller than optimal. It is impressive that with a small $\epsilon$, e.g., $\epsilon=1 \%$, the throughput of PASS is over $90 \%$ more than optimal, while in the same time the maximum delays of PASS are less than $331 \mathrm{~ms}$ which is still acceptable for video conferencing. In average, we observe a $2.0 \%$ throughput increment as compared to optimal, but with a $2.2 \%$ violation with the maximum delay constraints, when $\epsilon$ is decreased by $1 \%$ for instances where $\epsilon \leq 49 \%$.
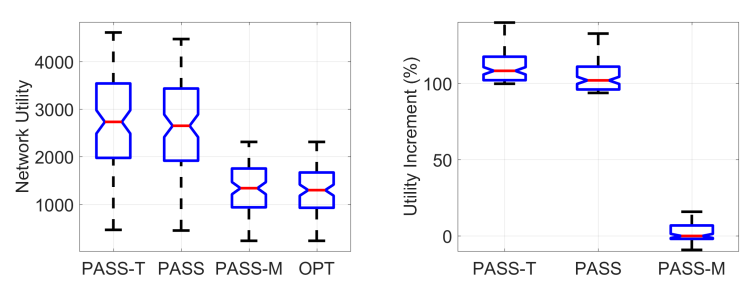

(a) Network utility results of different (b) Network utility increment comalgorithms, with $\epsilon=3 \%$ in PASS. pared to optimal, with $\epsilon=3 \%$ in PASS.

Figure 4: Simulation results of using PASS to maximize network utility, with $R_{1}=R_{2}=80$ and $D_{1}=D_{2}=150$.

\subsection{Use PASS to Maximize Network Utility}

Finally we use PASS to maximize aggregate user utilities, subject to link capacity constraints, maximum delay constraints, and throughput requirements (i.e., to solve MUDT with formula (1)). We assume the objective is (1a) where $\mathcal{U}_{i}^{t}\left(\left|f_{i}\right|\right)=w_{i} \cdot\left|f_{i}\right|, i=1,2$. And we assume $R_{1}=R_{2}=80$, and $D_{1}=D_{2}=150$ in the formula (1).

We vary the weight $w_{1}$ (resp. $w_{2}$ ) from 1 to 10 with a step of 1 , thus leading to 100 simulation instances each of which is characterized by a specific $\left\langle w_{1}, w_{2}\right\rangle, 1 \leq w_{1} \leq 10,1 \leq w_{2} \leq 10$. For each instance, we respectively run PASS, PASS-M, PASS-T, and compare their solutions with the optimal. Note that we obtain the optimal solution by solving multiple-unicast flow problems in the time-expanded network, similar to Sec. 5.1 and 5.2.

We present the achieved network utilities of different algorithms of the 100 simulation instances in Fig. 4(a). And in Fig. 4(b), we give the utility increment (\%) of our designed algorithms as compared to the optimal utility. Note that PASS, PASS-M, and PASS-T can obtain utilities that is strictly greater than optimal, because all of the three algorithms optimize utility subject to relaxed constraints, while the optimal utility is achieved by a feasible solution strictly meeting all the constraints.

From Fig. 4 we learn that PASS and PASS-T obtain a huge utility improvement compared to optimal (over $100 \%$ more than optimal), while the utility achieved by PASS-M is close-to-optimal. According to Thm. 3.2, theoretically PASS can violate both throughput requirements and maximum delay constraints. Empirically, (i) the throughput achieved by PASS is 138 (resp. 302) in average for the first unicast (resp. second unicast), both satisfying throughput requirements $R_{1}=R_{2}=80$. (ii) The maximum delay experienced by PASS is 195 (resp. 301) in average for the first unicast (resp. second unicast), both violating maximum delay constraints $D_{1}=D_{2}=150$. But considering that video conferencing applications can accept a delay less than $400 \mathrm{~ms}$ [12], the solution of PASS is acceptable. According to Thm. 3.3, theoretically PASS-M can meet maximum delay constraints while violate throughput requirements. Empirically, the throughput achieved by PASS-M is 71 (resp. 154) in average for the first unicast (resp. second unicast). It is clear that the first unicast flow violates throughput requirement. According to Thm. 3.4, theoretically PASS-T can meet throughput requirements while violate maximum delay constraints. Empirically, the maximum delay experienced by PASS-T is 222 (resp. 322) in average for the first unicast (resp. second unicast), both violating maximum delay constraints but within $400 \mathrm{~ms}$ that is the largest acceptable delay. 
Network Utility Maximization under Maximum Delay

Constraints and Throughput Requirements

\section{CONCLUSION}

We consider the problem of maximizing aggregate user utilities subject to link capacity constraints, maximum delay constraints, and throughput requirements. A user's utility is a concave function of the achieved throughput or the experienced maximum delay. The problem is uniquely challenging due to the need of jointly considering maximum delay constraints and throughput requirements. We first prove that it is NP-complete either (i) to construct a feasible solution meeting all constraints, or (ii) to obtain an optimal solution after we relax maximum delay constraints or throughput requirements up to constant ratios. We then design the first polynomialtime approximation algorithm named PASS to obtain solutions that (i) achieve constant or problem-dependent approximation ratios, at the cost of (ii) violating maximum delay constraints or throughput requirements up to constant or problem-dependent ratios, under realistic conditions. PASS is practically useful since our conditions are satisfied in many popular application settings. We evaluate PASS empirically using extensive simulations of routing delay-critical video-conferencing traffic over Amazon EC2 datacenters. Our design leverage a new understanding between maximum-delay-aware problems and their average-delay-aware counterparts, which can be of independent interest and suggest a new avenue for solving maximum-delay-aware network optimization problems.

\section{REFERENCES}

[1] Zizhong Cao, Paul Claisse, René-Jean Essiambre, Murali Kodialam, and TV Lakshman. 2017. Optimizing throughput in optical networks: The joint routing and power control problem. IEEE/ACM Trans. Networking 25, 1 (2017), 199-209.

[2] Xiangwen Chen, Minghua Chen, Baochun Li, Yao Zhao, Yunnan Wu, and Jin Li. 2011. Celerity: a low-delay multi-party conferencing solution. In Proc. ACM Int'l Conf. Multimedia. 493-502.

[3] Jose R Correa, Andreas S Schulz, and Nicolás E Stier Moses. 2004. Computational complexity, fairness, and the price of anarchy of the maximum latency problem. In Proc. Int'l Conf. Integer Programming and Combinatorial Optimization. 59-73.

[4] José R Correa, Andreas S Schulz, and Nicolás E Stier-Moses. 2007. Fast, fair, and efficient flows in networks. Operations Research 55, 2 (2007), 215-225.

[5] Fabrizio Devetak, Junghwan Shin, Tricha Anjali, and Sanjiv Kapoor. 2011. Minimizing path delay in multipath networks. In Proc. IEEE Int'l Conf. Communications. $1-5$.

[6] Lester R Ford and Delbert R Fulkerson. 1956. Maximal flow through a network. Canadian journal of Mathematics 8, 3 (1956), 399-404

[7] Benjamin Grimmer and Sanjiv Kapoor. 2016. Nash equilibrium and the price of anarchy in priority based network routing. In Proc. IEEE Int'l Conf. Computer Communications. 1-9.

[8] Martin Grötschel, László Lovász, and Alexander Schrijver. 2012. Geometric algorithms and combinatorial optimization. Springer Science \& Business Media.

[9] Mohammad H Hajiesmaili, Lok To Mak, Zhi Wang, Chuan Wu, Minghua Chen, and Ahmad Khonsari. 2017. Cost-Effective Low-Delay Design for Multiparty Cloud Video Conferencing. IEEE Trans. Multimedia 19, 12 (2017), 2760-2774.

[10] Chi-Yao Hong, Srikanth Kandula, Ratul Mahajan, Ming Zhang, Vijay Gill, Mohan Nanduri, and Roger Wattenhofer. [n. d.]. Achieving high utilization with softwaredriven WAN. In Proc. ACM SIGCOMM Computer Communication Review.

[11] IBM. 2017. Cplex Optimizer. (2017). Available at https://www-01.ibm.com/ software/commerce/optimization/cplex-optimizer/.

[12] ITU. 2003. SERIES G: TRANSMISSION SYSTEMS AND MEDIA, DIGITAL SYS TEMS AND NETWORKS. (2003).

[13] Sushant Jain, Alok Kumar, Subhasree Mandal, Joon Ong, Leon Poutievski, Arjun Singh, Subbaiah Venkata, Jim Wanderer, Junlan Zhou, Min Zhu, et al. [n. d.] B4: Experience with a globally-deployed software defined WAN. In Proc. ACM SIGCOMM Computer Communication Review.

[14] Frank P Kelly, Aman K Maulloo, and David KH Tan. 1998. Rate control for communication networks: shadow prices, proportional fairness and stability Journal of the Operational Research society 49, 3 (1998), 237-252.

[15] Qingyu Liu, Lei Deng, Haibo Zeng, and Minghua Chen. 2018. A Tale of Two Metrics in Network Delay Optimization. In Proc. IEEE Int'l Conf. Computer Communications. 2123-2131.

[16] Yinan Liu, Di Niu, and Baochun Li. 2016. Delay-optimized video traffic routing in software-defined interdatacenter networks. IEEE Trans. Multimedia 18, 5 (2016), 865-878.
Submission to MobiHoc '19, July 2-5, 2019, Catania, Italy

[17] Steven H Low and David E Lapsley. 1999. Optimization flow controlâĂŤI: basic algorithm and convergence. IEEE/ACM Trans. Networking 7, 6 (1999), 861-874.

[18] Satyajayant Misra, Guoliang Xue, and Dejun Yang. 2009. Polynomial time approximations for multi-path routing with bandwidth and delay constraints. In Proc. IEEE Int'l Conf. Computer Communications. 558-566.

[19] Daniel Pérez Palomar and Mung Chiang. 2006. A tutorial on decomposition methods for network utility maximization. IEEE Journal on Selected Areas in Communications 24, 8 (2006), 1439-1451.

[20] Florian Potra and Yinyu Ye. 1993. A quadratically convergent polynomial algorithm for solving entropy optimization problems. SIAM fournal on Optimization 3, 4 (1993), 843-860.

[21] Jiantao Wang, Lun Li, Steven H Low, and John C Doyle. 2003. Can shortest-path routing and TCP maximize utility. In Proc. IEEE Int'l Conf. Computer Communications. 2049-2056.

[22] Ira M Weinstein. 2008. PolycomâĂŹs Lost Packet Recovery (LPR) Capability. Wainhouse Research (2008).

[23] WIKI. [n. d.]. Polynomial time approximation scheme. ([n. d.]). Available at https://en.wikipedia.org/wiki/Polynomial-time_approximation_scheme.

[24] Yinyu Ye. 1991. An $O\left(n^{3} L\right)$ potential reduction algorithm for linear programming. Mathematical programming 50, 1-3 (1991), 239-258.

[25] Ruozhou Yu, Guoliang Xue, and Xiang Zhang. 2018. Application Provisioning in FOG Computing-enabled Internet-of-Things: A Network Perspective. In Proc. IEEE Int'l Conf. Computer Communications. 783-791.

[26] Weiyi Zhang, Jian Tang, Chonggang Wang, and Shanaka de Soysa. 2010. Reliable adaptive multipath provisioning with bandwidth and differential delay constraints. In Proc. IEEE Int'l Conf. Computer Communications. 1-9.

\section{APPENDIX}

\subsection{Proof to Lem. 3.1}

Proof. According to Algorithm 1 , for any $i=1,2, \ldots, K, \bar{f}_{i}$ is obtained by iteratively deleting $\epsilon \cdot\left|\hat{f}_{i}\right|$ rate from $\hat{f}_{i}$. Suppose that there are in total $N_{i}$ iterations to get $\bar{f}_{i}$ by deleting rate from $\hat{f}_{i}$ (namely assume $N_{i}$ to be the number of iterations of the while-loop of line 8). And we use $f_{i}^{n}$ to represent the flow of the unicast $i$ at the beginning of the $n$-th iteration (or equivalently, at the end of the $(n-1)$-th iteration). Obviously, $f_{i}^{1}=\hat{f}_{i}, f_{i}^{N_{i}+1}=\bar{f}_{i}$. We denote $P_{i}^{n}$ as the set of of all flow-carrying paths in flow $f_{i}^{n}$, and $p_{i}^{n} \in P_{i}^{n}$ as the slowest flow-carrying path in $P_{i}^{n}$. In the $n$-th iteration of the unicast $i$, PASS delete some rate, say $x_{i}^{n}>0$, from $p_{i}^{n}$.

Since all link delays are non-negative constants, the path delay cannot increase with reduced flow rate. Thus,

$$
\mathcal{M}\left(f_{i}^{n+1}\right) \leq \mathcal{M}\left(f_{i}^{n}\right), \forall n=1,2, \ldots, N_{i}, \forall i=1,2, \ldots, K .
$$

Considering the total delay of the unicast $i$, for any $1 \leq n \leq N_{i}$, we have the following held for any $i=1,2, \ldots, K$

$$
\begin{aligned}
& \mathcal{T}\left(f_{i}^{n}\right)=\sum_{e \in E: e \notin p_{i}^{n}}\left[x_{i}^{e} d_{e}\right]+\sum_{e \in E: e \in p_{i}^{n}}\left[x_{i}^{e} d_{e}\right] \\
& =\sum_{e \in E: e \notin p_{i}^{n}}\left[x_{i}^{e} d_{e}\right]+\sum_{e \in E: e \in p_{i}^{n}}\left[\left(x_{i}^{e}-x_{i}^{n}\right) d_{e}+x_{i}^{n} d_{e}\right] \\
& \stackrel{(a)}{=} \sum_{e \in E: e \notin p_{i}^{n}}\left[x_{i}^{e} d_{e}\right]+\sum_{e \in E: e \in p_{i}^{n}}\left[\left(x_{i}^{e}-x_{i}^{n}\right) d_{e}\right]+x_{i}^{n} \mathcal{M}\left(f_{i}^{n}\right) \\
& \stackrel{(b)}{=} \mathcal{T}\left(f_{i}^{n+1}\right)+x_{i}^{n} \mathcal{M}\left(f_{i}^{n}\right) \stackrel{(c)}{\geq} \mathcal{T}\left(f_{i}^{n+1}\right)+x_{i}^{n} \mathcal{M}\left(\bar{f}_{i}\right) .
\end{aligned}
$$

In (18), equality ( $a$ ) holds because $\sum_{e \in p_{i}^{n}} d_{e}$ is the path delay of the slowest flow-carrying path $p_{i}^{n}$. Equality $(b)$ holds because flow $f_{i}^{n+1}$ is the flow when $f_{i}^{n}$ deletes $x_{i}^{n}$ rate from path $p_{i}^{n}$. Inequality (c) comes from (17) and $f_{i}^{N_{i}+1}=\bar{f}_{i}$.

We then do summation for (18) over $n \in\left[1, N_{i}\right]$, and get

$$
\mathcal{T}\left[\hat{f}_{i}\right]=\mathcal{T}\left(f_{i}^{1}\right) \geq \mathcal{T}\left(f_{i}^{N_{i}+1}\right)+\left(\sum_{n=1}^{N_{i}} x_{i}^{n}\right) \cdot \mathcal{M}\left(\bar{f}_{i}\right)
$$




$$
=\mathcal{T}\left[\bar{f}_{i}\right]+\epsilon \cdot\left|\hat{f}_{i}\right| \cdot \mathcal{M}\left(\bar{f}_{i}\right),
$$

which proves our lemma.

\subsection{Proof to Thm. 3.2}

Proof. First, we prove the polynomial time complexity. Due to condition 1, both problem (2) and (3) can be solved in polynomial time, since (i) they are convex programs with a polynomial number of variables and a polynomial number of constraints, and (ii) convex programming problems can be solved up to an arbitrarily small additive error in polynomial time (e.g., see $[8,20]$ for details). For example, the time complexity is $O\left(|E|^{3} K^{3} \mathcal{L}\right)$ where $\mathcal{L}$ is the input size of the instance of the problem (2) or (3) if they are linear programs [24]. After solving the average-delay-aware problem, we get $K$ single-unicast flows each of which is defined on edges. By the classic flow decomposition technique [6], we can then achieve $K$ single-unicast flows $\hat{f}=\left\{\hat{f}_{i}, i=1,2, \ldots, K\right\}$ each of which is defined on paths within a time of $O\left(|V|^{2}|E| K\right)$. Note that the flow decomposition outputs at most $|E|$ paths for each $\hat{f}_{i}$, and hence there are at most $|E|$ iterations to obtain each $\bar{f}_{i}$ by deleting rate from $\hat{f}_{i}$. Overall, Algorithm 1 has a polynomial time complexity that is even independent to $\epsilon$ when all conditions are satisfied.

Second, we prove the existence of $\bar{f}$.

(i) Suppose (1a) is the objective of the problem (1). Because problem (1) is feasible and $f^{*}$ is its optimal solution, $f^{*}$ must satisfy all the constraints of problem (1), implying that $f^{*}$ also satisfies the constraints $(2 \mathrm{~b})$ and $(2 \mathrm{~d})$ of the problem (2) that is the averagedelay-aware counterpart of the problem (1). Now consider that we have $\mathcal{T}(g) \leq \mathcal{M}(g) \cdot|g|$ for any single-unicast flow $g$, for any $i=1,2, \ldots, K$, the following holds

$$
\mathcal{T}\left(f_{i}^{*}\right) \leq \mathcal{M}\left(f_{i}^{*}\right) \cdot\left|f_{i}^{*}\right| \stackrel{(a)}{\leq} D_{i} \cdot\left|f_{i}^{*}\right|,
$$

where the inequality (a) comes from that $f^{*}$ meets the constraints (1d) of the problem (1). Therefore, $f^{*}$ is also a feasible solution to the problem (2). Due to the existence of $f^{*}$, problem (2) must be feasible and hence Algorithm 1 must return a solution $\bar{f}$.

(ii) Suppose (1b) is the objective of the problem (1). Because problem (1) is feasible and $f^{*}$ is its optimal solution, $f^{*}$ must meet all the constraints of problem (1), e.g., we have $\left|f_{i}^{*}\right| \geq R_{i}, \forall i=1,2, \ldots, K$. Now we construct another network flow $f$ based on $f^{*}$ as follows: for each $i=1,2, \ldots, K$, we obtain $f_{i}$ directly from $f_{i}^{*}$, by deleting flow rate from arbitrary flow-carrying paths of $f_{i}^{*}$ till $\left|f_{i}^{*}\right|=R_{i}$. The existence of $f^{*}$ implies the existence of $f$. For problem (3), it is clear that $f$ meets the throughput requirements (3b). Since $f^{*}$ meets the constraint (1e), $f$ must satisfy the constraint (3d). Since we delete certain flow rate from $f_{i}^{*}$ to obtain $f_{i}$, it is clear that the maximum delay does not increase, i.e., we have

$$
\mathcal{M}\left(f_{i}\right) \leq \mathcal{M}\left(f_{i}^{*}\right), \forall i=1,2, \ldots, K,
$$

further implying the following for any $i=1,2, \ldots, K$

$$
\mathcal{T}\left(f_{i}\right) \leq \mathcal{M}\left(f_{i}\right) \cdot\left|f_{i}\right|=\mathcal{M}\left(f_{i}\right) \cdot R_{i} \leq \mathcal{M}\left(f_{i}^{*}\right) \cdot R_{i} \leq D_{i} \cdot R_{i},
$$

i.e., $f$ meets the constraints (3c). Therefore, $f$ is a feasible solution to the problem (3). Due to the existence of $f$, problem (3) must be feasible and hence Algorithm 1 must return a solution $\bar{f}$.

Third, we prove that $\bar{f}$ satisfies the relaxed constraints (5). Suppose $\hat{f}$ is the solution to the average-delay-aware problem in line 5 .
Then clearly that $\hat{f}$ meets the following constraints:

$$
\begin{gathered}
\left|\hat{f}_{i}\right| \geq R_{i}, \forall i=1,2, \ldots, K, \\
\mathcal{A}\left(\hat{f}_{i}\right) \leq D_{i}, \forall i=1,2, \ldots, K, \\
\hat{f}=\left\{\hat{f}_{1}, \hat{f}_{2}, \ldots, \hat{f}_{K}\right\} \in \mathcal{X} .
\end{gathered}
$$

We know $\bar{f}_{i}$ is the solution by deleting a rate of $\epsilon \cdot\left|\hat{f}_{i}\right|$ from $\hat{f}_{i}$ for each $i=1,2, \ldots, K$. It is clear that $\bar{f}$ satisfies the constraints (5a) and (5c). Now we look at the constraints (5b).

According to our Lem. 3.1, for any $i=1,2, \ldots, K$, it holds that

$$
\epsilon \cdot\left|\hat{f}_{i}\right| \cdot \mathcal{M}\left(\bar{f}_{i}\right) \leq \mathcal{T}\left(\hat{f}_{i}\right)-\mathcal{T}\left(\bar{f}_{i}\right) \leq \mathcal{T}\left(\hat{f}_{i}\right),
$$

implying that $\mathcal{M}\left(\bar{f}_{i}\right) \leq \mathcal{A}\left(\hat{f}_{i}\right) / \epsilon, \forall i=1,2, \ldots, K$. Based on the satisfied constraints (20b), we have the following for any $i=1,2, \ldots, K$

$$
\mathcal{M}\left(\bar{f}_{i}\right) \leq \mathcal{A}\left(\hat{f}_{i}\right) / \epsilon \leq D_{i} / \epsilon .
$$

Finally, we prove the approximation ratio of $\bar{f}$. If (1a) is the objective of problem (1), we have

$$
\begin{aligned}
& \sum_{i=1}^{K} \mathcal{U}_{i}^{t}\left(\left|\bar{f}_{i}\right|\right)=\sum_{i=1}^{K} \mathcal{U}_{i}^{t}\left((1-\epsilon) \cdot\left|\hat{f}_{i}\right|\right) \\
& \stackrel{(a)}{\geq}(1-\epsilon) \cdot \sum_{i=1}^{K} \mathcal{U}_{i}^{t}\left(\left|\hat{f}_{i}\right|\right) \stackrel{(b)}{\geq}(1-\epsilon) \cdot \sum_{i=1}^{K} \mathcal{U}_{i}^{t}\left(\left|f_{i}^{*}\right|\right)
\end{aligned}
$$

where the inequality (b) holds because in the second part of this proof, we have proved that $f^{*}$ is a feasible solution to the averagedelay-aware problem (2), while $\hat{f}$ is its optimal solution. Inequality (a) comes from the following inequalities for each $i=1,2, \ldots, K$

$$
\begin{aligned}
& \mathcal{U}_{i}^{t}\left((1-\epsilon) \cdot\left|\hat{f}_{i}\right|\right)=\mathcal{U}_{i}^{t}\left(\epsilon \cdot 0+(1-\epsilon) \cdot\left|\hat{f}_{i}\right|\right) \\
& \stackrel{(c)}{\geq} \epsilon \cdot \mathcal{U}_{i}^{t}(0)+(1-\epsilon) \cdot \mathcal{U}_{i}^{t}\left(\left|\hat{f}_{i}\right|\right) \stackrel{(d)}{\geq}(1-\epsilon) \cdot \mathcal{U}_{i}^{t}\left(\left|\hat{f}_{i}\right|\right),
\end{aligned}
$$

where the inequality (c) holds due to the concavity of the function $\mathcal{U}_{i}^{t}(\cdot)$, and the inequality (d) comes from that the function $\mathcal{U}_{i}^{t}(\cdot)$ is non-negative, considering that the condition 1 is satisfied.

If (1b) is the objective of problem (1), we assume $f$ is the feasible solution to the average-delay-aware problem (3) that is constructed from $f^{*}$ as discussed in the second part of this proof. Then we have

$$
\begin{aligned}
& \sum_{i=1}^{K} \mathcal{U}_{i}^{d}\left(\mathcal{M}\left(\bar{f}_{i}\right)\right) \leq \sum_{i=1}^{K} \mathcal{U}_{i}^{d}\left(\mathcal{A}\left(\hat{f}_{i}\right) / \epsilon\right) \\
& \stackrel{(a)}{\leq} \frac{1}{\epsilon} \cdot \sum_{i=1}^{K} \mathcal{U}_{i}^{d}\left(\mathcal{A}\left(\hat{f}_{i}\right)\right) \stackrel{(b)}{\leq} \frac{1}{\epsilon} \cdot \sum_{i=1}^{K} \mathcal{U}_{i}^{d}\left(\mathcal{A}\left(f_{i}\right)\right) \\
& \leq \frac{1}{\epsilon} \cdot \sum_{i=1}^{K} \mathcal{U}_{i}^{d}\left(\mathcal{M}\left(f_{i}\right)\right) \stackrel{(c)}{\leq} \frac{1}{\epsilon} \cdot \sum_{i=1}^{K} \mathcal{U}_{i}^{d}\left(\mathcal{M}\left(f_{i}^{*}\right)\right),
\end{aligned}
$$

where the inequality (a) comes from the satisfied condition 2, the inequality (b) holds since $f$ is feasible to problem (3) while $\hat{f}$ is optimal to problem (3), and the inequality (c) is true because of the inequality (19) and the non-decreasing property of $\mathcal{U}_{i}^{d}(\cdot)$. 
Network Utility Maximization under Maximum Delay

Constraints and Throughput Requirements

\subsection{Proof to Thm. 2.1}

Proof. First, we consider the following problem that is a special case of the MUDT with relaxed maximum delay constraints,

$$
\begin{array}{cl}
\max & -\mathcal{M}\left(f_{1}\right) \\
\text { s.t. } & \left|f_{1}\right| \geq R_{1}, \\
& \mathcal{M}\left(f_{1}\right) \leq+\infty, \\
& f=\left\{f_{1}\right\} \in \mathcal{X} .
\end{array}
$$

It has been proved to be NP-complete to find the optimal solution to above problem (see Appendix of [18]).

Second, we consider the following problem that is a special case of the MUDT with relaxed throughput requirements,

$$
\begin{array}{cl}
\max & \left|f_{1}\right| \\
\text { s.t. } & \left|f_{1}\right| \geq 0, \\
& \mathcal{M}\left(f_{1}\right) \leq D_{1}, \\
& f=\left\{f_{1}\right\} \in \mathcal{X} .
\end{array}
$$

Follow a similar proof as that in the Appendix of [18], it can be proved to be NP-complete to find the optimal solution to the aforementioned problem.

Third, also following a similar proof as that in the Appendix of [18], it can be proved that it is NP-complete even to construct a feasible solution to the following problem that is a special case of our MUDT, strictly meeting all constraints

$$
\begin{array}{cl}
\max & \mathcal{U}_{1}^{t}\left(\left|f_{1}\right|\right) \\
\text { s.t. } & \left|f_{1}\right| \geq R_{1}, \\
& \mathcal{M}\left(f_{1}\right) \leq D_{1}, \\
& f=\left\{f_{1}\right\} \in \mathcal{X},
\end{array}
$$

where $\mathcal{U}_{1}^{t}\left(\left|f_{1}\right|\right)=1$ which is a constant.

\subsection{Proof to Thm. 3.3}

Proof. First, due to the same proof to Thm. 3.2, Algorithm 2 has a polynomial time complexity, and must give a solution $\bar{f}$.

Second, it is straightforward that constraints (8b) and (8c) are met. Now let us denote $\left(\left|\hat{f}_{i}\right|-\left|\bar{f}_{i}\right|\right) /\left|\hat{f}_{i}\right|$ as $\epsilon_{i}$. Thus $\epsilon_{\min } \leq \epsilon_{i} \leq \epsilon_{\max }$ for any $i=1,2, \ldots, K$, implying the following

$$
\left|\bar{f}_{i}\right|=\left(1-\epsilon_{i}\right) \cdot\left|\hat{f}_{i}\right| \geq\left(1-\epsilon_{\max }\right) \cdot\left|\hat{f}_{i}\right|, \forall i=1,2, \ldots, K,
$$

i.e., the constraints $(8 \mathrm{~b})$ are satisfied.

Third, following the same proof as to Thm. 3.2, the approximation ratio (9) can be proved.

As for the approximation ratio (10), let as assume $\tilde{f}$ to be the solution where for each $i=1,2, \ldots, K$, we delete $\epsilon_{\min }\left|\hat{f}_{i}\right|$ rate from the slowest flow-carrying paths of $\hat{f}_{i}$ to obtain $\tilde{f}_{i}$. It is clear that

$$
\mathcal{M}\left(\bar{f}_{i}\right) \leq \mathcal{M}\left(\tilde{f}_{i}\right), \forall i=1,2, \ldots, K,
$$

because both $\bar{f}_{i}$ and $\tilde{f}_{i}$ are flows after we delete rates from the slowest flow-carrying paths of $\hat{f}_{i}$, but the amount of deleted rate to obtain $\bar{f}_{i}$ is no smaller than the amount of deleted rate to obtain $\tilde{f}_{i}$, for each $i=1,2, \ldots, K$. Therefore, we have the following

$$
\sum_{i=1}^{K} \mathcal{U}_{i}^{d}\left(\mathcal{M}\left(\bar{f}_{i}\right)\right) \leq \sum_{i=1}^{K} \mathcal{U}_{i}^{d}\left(\mathcal{M}\left(\tilde{f}_{i}\right)\right) \stackrel{(a)}{\leq} \frac{1}{\epsilon_{\min }} \cdot \sum_{i=1}^{K} \mathcal{U}_{i}^{d}\left(\mathcal{M}\left(f_{i}^{*}\right)\right),
$$

Submission to MobiHoc '19, July 2-5, 2019, Catania, Italy

where the inequality (a) comes from our Thm. 3.2 , since $\tilde{f}$ is also the solution returned if we use Algorithm 1 with $\epsilon=\epsilon_{\min }$ to solve the problem (1).

\subsection{Proof to Thm. 3.4}

Proof. Same to the proof as that of Thm. 3.2, it holds that PASS-T must return a solution $\bar{f}$ in a polynomial time, meeting the constraints (11a), (11c), and providing the approximation ratio (12).

Because that $\bar{g}$ is the solution of PASS, we have

$$
\begin{aligned}
& \mathcal{M}\left(\bar{g}_{i}\right) \leq D_{i} / \epsilon, \forall i=1,2, \ldots, K, \\
& \sum_{i=1}^{K} \mathcal{U}_{i}^{d}\left(\mathcal{M}\left(\bar{g}_{i}\right)\right) \leq \frac{1}{\epsilon} \cdot \sum_{i=1}^{K} \mathcal{U}_{i}^{d}\left(\mathcal{M}\left(f_{i}^{*}\right)\right) .
\end{aligned}
$$

According to the definition of $\lambda$, we have

$$
\mathcal{M}\left(\bar{f}_{i}\right) \leq \lambda \cdot \mathcal{M}\left(\bar{g}_{i}\right), \forall i=1,2, \ldots, K,
$$

implying the following considering the inequality (22)

$$
\mathcal{M}\left(\bar{f}_{i}\right) \leq \lambda \cdot D_{i} / \epsilon, \forall i=1,2, \ldots, K,
$$

i.e., $\bar{f}$ satisfies the constraints (11b). We further have

$$
\begin{aligned}
& \sum_{i=1}^{K} \mathcal{U}_{i}^{d}\left(\mathcal{M}\left(\bar{f}_{i}\right)\right) \leq \sum_{i=1}^{K} \mathcal{U}_{i}^{d}\left(\lambda \cdot \mathcal{M}\left(\bar{g}_{i}\right)\right) \\
& \stackrel{(a)}{\leq} \lambda \cdot \sum_{i=1}^{K} \mathcal{U}_{i}^{d}\left(\mathcal{M}\left(\bar{g}_{i}\right)\right) \stackrel{(b)}{\leq} \frac{\lambda}{\epsilon} \cdot \sum_{i=1}^{K} \mathcal{U}_{i}^{d}\left(\mathcal{M}\left(f_{i}^{*}\right)\right),
\end{aligned}
$$

where the inequality (a) comes from the satisfied condition 2 , and the inequality (b) holds due to the inequality (23). Thus the approximation ratio (13) holds. 